\title{
Determinant expressions for $q$-harmonic congruences and degenerate Bernoulli numbers
}

\author{
Karl Dilcher* \\ Department of Mathematics and Statistics \\ Dalhousie University, Halifax, NS, B3H 3J5, Canada \\ dilcher@mathstat.dal.ca
}

Submitted: Feb 16, 2008; Accepted: Apr 17, 2008; Published: Apr 28, 2008

Mathematics Subject Classification: 11B65, 11B68

\begin{abstract}
The generalized harmonic numbers $H_{n}^{(k)}=\sum_{j=1}^{n} j^{-k}$ satisfy the well-known congruence $H_{p-1}^{(k)} \equiv 0(\bmod p)$ for all primes $p \geq 3$ and integers $k \geq 1$. We derive $q$-analogs of this congruence for two different $q$-analogs of the sum $H_{n}^{(k)}$. The results can be written in terms of certain determinants of binomial coefficients which have interesting properties in their own right. Furthermore, it is shown that one of the classes of determinants is closely related to degenerate Bernoulli numbers, and new properties of these numbers are obtained as a consequence.
\end{abstract}

\section{Introduction}

The harmonic numbers $H_{n}$ are defined by

$$
H_{n}:=\sum_{j=1}^{n} \frac{1}{j}, \quad n \geq 0,
$$

where by convention $H_{0}=0$. These numbers have been studied extensively (see, e.g., [10, p. $272 \mathrm{ff}$.$] ), and they have important applications in combinatorics, number theory, and$ the analysis of algorithms. The harmonic numbers have also been generalized in various different ways; for a recent summary of generalizations, see [5]. In this paper we will be concerned with the generalized harmonic numbers defined by

$$
H_{n}^{(k)}:=\sum_{j=1}^{n} \frac{1}{j^{k}}, \quad n \geq 0,
$$

\footnotetext{
* Supported in part by the Natural Sciences and Engineering Research Council of Canada
} 
where we restrict our attention to positive integer parameters $k$. Obviously, $H_{n}^{(1)}=H_{n}$, and $\lim _{n \rightarrow \infty} H_{n}^{(k)}=\zeta(k)$ for $k \geq 2$, where $\zeta(k)$ is the Riemann zeta function. See, e.g., [10] for some further properties.

Many special functions, sequences, and identities have interesting and meaningful $q$-analogs. For a general discussion of $q$-series, $q$-analogs, and their importance in combinatorics, analysis, number theory, and other areas, see, e.g., [2, Ch. 10-12]. A q-analog of $H_{n}$ is given by the $q$-harmonic numbers

$$
H_{n}(q):=\sum_{j=1}^{n} \frac{1}{[j]_{q}}, \quad n \geq 0, \quad|q|<1,
$$

where

$$
[j]_{q}:=\frac{1-q^{j}}{1-q}=1+q+\ldots+q^{j-1},
$$

A different $q$-analog of $H_{n}$ is

$$
\widetilde{H}_{n}(q):=\sum_{j=1}^{n} \frac{q^{j}}{[j]_{q}} .
$$

One of the most remarkable properties of the harmonic numbers is the congruence of Wolstenholme [17] which states that for primes $p \geq 5$,

$$
H_{p-1} \equiv 0 \quad\left(\bmod p^{2}\right)
$$

This, by the way, is closely related to another famous congruence due to Wolstenhome, namely

$$
\left(\begin{array}{c}
2 p-1 \\
p-1
\end{array}\right) \equiv 1 \quad\left(\bmod p^{3}\right) .
$$

for primes $p \geq 5$. See, e.g., [1] for this connection, and [14] for a well-known conjecture related to this.

Andrews [1] proved a $q$-analog of the weaker version $(\bmod p)$ of the congruence $(1.2)$, namely

$$
H_{p-1}(q) \equiv \frac{p-1}{2}(1-q) \quad\left(\bmod [p]_{q}\right)
$$

as well as

$$
\widetilde{H}_{p-1}(q) \equiv-\frac{p-1}{2}(1-q) \quad\left(\bmod [p]_{q}\right),
$$

for primes $p \geq 3$. More recently Shi and Pan [16] extended (1.3) to

$$
H_{p-1}(q) \equiv \frac{p-1}{2}(1-q)+\frac{p^{2}-1}{24}(1-q)^{2}[p]_{q} \quad\left(\bmod [p]_{q}^{2}\right),
$$

for primes $p \geq 5$. These congruences, and all others to come, are to be understood as congruences in the polynomial ring $\mathbb{Z}[q]$. Note that by $(1.1)$ it is clear that $[p]_{q}$, as $p$ th cyclotomic polynomial, is irreducible; hence the demominator of $H_{p-1}(q)$, seen as a rational function of $q$, is relatively prime to $[p]_{q}$. 
As far as the generalized harmonic numbers are concerned, the analog of Wolstenholme's congruence (1.2) is in general true only modulo $p$. In fact, Glaisher [8] showed that

$$
H_{p-1}^{(k)} \equiv 0 \quad(\bmod p)
$$

for all integers $k \geq 1$. A little later, Glaisher himself proved refinements of this congruence; see [9] or [13, p. 353].

It is the main purpose of this paper to find $q$-analogs of the congruence (1.5). We will derive congruences $\left(\bmod [p]_{q}\right)$ for the generalized (or higher-order) $q$-harmonic numbers

$$
H_{n}^{(k)}(q):=\sum_{j=1}^{n} \frac{1}{[j]_{q}^{k}}
$$

and

$$
\widetilde{H}_{n}^{(k)}(q):=\sum_{j=1}^{n} \frac{q^{j}}{[j]_{q}^{k}},
$$

for all integers $k \geq 1$. The case $k=1$ is given by (1.3) and (1.4), and the lemma in [16] states that

$$
\begin{aligned}
& H_{p-1}^{(2)}(q) \equiv-\frac{(p-1)(p-5)}{12}(1-q)^{2} \quad\left(\bmod [p]_{q}\right) \\
& \widetilde{H}_{p-1}^{(2)}(q) \equiv-\frac{p^{2}-1}{12}(1-q)^{2} \quad\left(\bmod [p]_{q}\right) .
\end{aligned}
$$

The main results of this paper will be presented in the next section, followed by their proofs in Sections 3 and 4. In Section 5 we study some properties of the polynomials in $p$ that occur in these results, and a connection with degenerate Bernoulli numbers is investigated in Section 6.

\section{The Main Results}

In terms of the $q$-analog of the congruence (1.5) it turns out that the sum $\widetilde{H}_{n}^{(k)}(q)$ is a somewhat more natural $q$-extension of $H_{n}^{(k)}$ than is $H_{n}^{(k)}(q)$. We therefore begin with a result concerning the former sum.

For any integer $k \geq 1$ we define the following determinant of binomial coefficients:

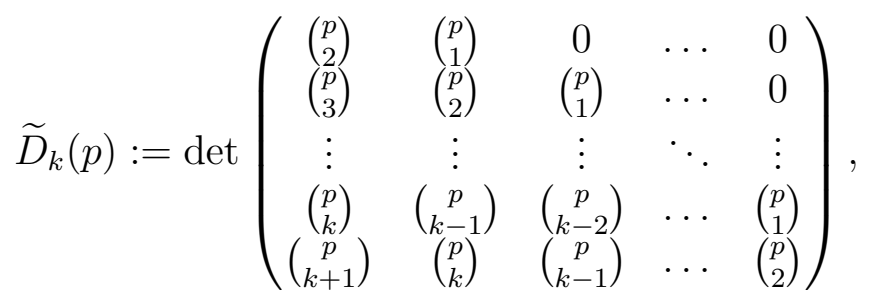

so that in particular

$$
\widetilde{D}_{1}(p)=\operatorname{det}\left(\left(\begin{array}{l}
p \\
2
\end{array}\right)\right)=\frac{p(p-1)}{2}
$$


and

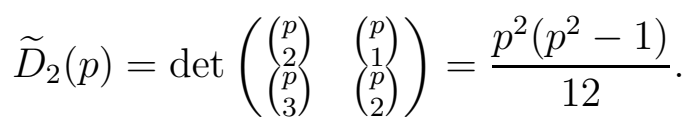

The fact that $-p^{-1} \widetilde{D}_{1}(p)$ and $-p^{-2} \widetilde{D}_{2}(p)$ occur in (1.4) and (1.9), respectively, is no coincidence. In fact, we have the following result.

Theorem 1. If $p \geq 3$ is a prime, then for all integers $k \geq 1$ we have

$$
\widetilde{H}_{p-1}^{(k)}(q) \equiv-\frac{1}{p^{k}} \widetilde{D}_{k}(p)(1-q)^{k} \quad\left(\bmod [p]_{q}\right)
$$

The first few determinants $\widetilde{D}_{k}(p)$ are listed in Table 1 below.

For our second main result, concerning the other type, $H_{n}^{(k)}(q)$, of $q$-harmonic sums, we define the determinants

$$
D_{k}(p):=\operatorname{det}\left(\begin{array}{ccccc}
\left(\begin{array}{c}
p+1 \\
2
\end{array}\right) & p & 0 & \ldots & 0 \\
\left(\begin{array}{c}
p+1 \\
3
\end{array}\right) & \left(\begin{array}{c}
p+1 \\
2
\end{array}\right) & p & \ldots & 0 \\
\vdots & \vdots & \vdots & \ddots & \vdots \\
\left(\begin{array}{c}
p+1 \\
k
\end{array}\right) & \left(\begin{array}{c}
p+1 \\
k-1
\end{array}\right) & \left(\begin{array}{c}
p+1 \\
k-2
\end{array}\right) & \ldots & p \\
\left(\begin{array}{l}
p+1 \\
k+1
\end{array}\right) & \left(\begin{array}{c}
p+1 \\
k
\end{array}\right) & \left(\begin{array}{c}
p+1 \\
k-1
\end{array}\right) & \ldots & \left(\begin{array}{c}
p+1 \\
2
\end{array}\right)
\end{array}\right) .
$$

In analogy to (2.2) and (2.3) we have

$$
D_{1}(p)=\operatorname{det}\left(\left(\begin{array}{c}
p+1 \\
2
\end{array}\right)\right)=\frac{p(p+1)}{2}
$$

and

$$
\left.D_{2}(p)=\operatorname{det}\left(\begin{array}{cc}
p+1 \\
2 \\
2+1 \\
3
\end{array}\right) \quad \begin{array}{c}
p \\
\left(\begin{array}{c}
p+1 \\
2
\end{array}\right)
\end{array}\right)=\frac{p^{2}(p+1)(p+5)}{12},
$$

and as before we see that $-p^{-1} D_{1}(-p)$ and $-p^{-2} D_{2}(-p)$ occur in (1.3) and (1.8), respectively. In fact, we have

Theorem 2. If $p \geq 3$ is a prime, then for all integers $k \geq 1$ we have

$$
H_{p-1}^{(k)}(q) \equiv \frac{(-1)^{k-1}}{p^{k}} D_{k}(-p)(1-q)^{k} \quad\left(\bmod [p]_{q}\right)
$$

Note that in contrast to (2.4) the argument of the polynomials $D_{k}$ is $-p$. The first few determinants $D_{k}(p)$ are listed in Table 2 below. 


\begin{tabular}{|r||r|l|}
\hline$k$ & $\widetilde{d}_{k}$ & $\widetilde{A}_{k}(p)$ \\
\hline 1 & 2 & $p-1$ \\
2 & 12 & $p^{2}-1$ \\
3 & 24 & $p^{2}-1$ \\
4 & 720 & $-\left(p^{2}-1\right)\left(p^{2}-19\right)$ \\
5 & 480 & $-\left(p^{2}-1\right)\left(p^{2}-9\right)$ \\
6 & 60480 & $\left(p^{2}-1\right)\left(2 p^{4}-145 p^{2}+863\right)$ \\
7 & 24192 & $\left(p^{2}-1\right)\left(p^{2}-25\right)\left(2 p^{2}-11\right)$ \\
8 & 3628800 & $-\left(p^{2}-1\right)\left(3 p^{6}-497 p^{4}+9247 p^{2}-33953\right)$ \\
9 & 1036800 & $-\left(p^{2}-1\right)\left(p^{2}-49\right)\left(3 p^{4}-50 p^{2}+167\right)$ \\
10 & 479001600 & $\left(p^{2}-1\right)\left(10 p^{8}-2993 p^{6}+114597 p^{4}-1184767 p^{2}+3250433\right)$ \\
11 & 21288960 & $\left(p^{2}-1\right)\left(p^{2}-9\right)\left(p^{2}-81\right)\left(2 p^{4}-49 p^{2}+173\right)$ \\
\hline
\end{tabular}

Table 1: $\widetilde{D}_{k}(p)=\widetilde{d}_{k}^{-1} p^{k} \widetilde{A}_{k}(p), k=1, \ldots, 11$.

\begin{tabular}{|r||r|l|}
\hline$k$ & $d_{k}$ & $A_{k}(p)$ \\
\hline 1 & 2 & 1 \\
2 & 12 & $p+5$ \\
3 & 8 & $p+3$ \\
4 & 720 & $-\left(p^{3}-p^{2}-109 p-251\right)$ \\
5 & 288 & $-(p+5)\left(p^{2}-6 p-19\right)$ \\
6 & 60480 & $\left.2 p^{5}-2 p^{4}-355 p^{3}+355 p^{2}+11153 p+19087\right)$ \\
7 & 17280 & $(p+7)\left(2 p^{4}-16 p^{3}-33 p^{2}+376 p+751\right)$ \\
8 & 3628800 & $-\left(3 p^{7}-3 p^{6}-917 p^{5}+917 p^{4}+39697 p^{3}-39697 p^{2}\right.$ \\
& & $-744383 p-1070017)$ \\
9 & 268800 & $-(p+3)(p+9)\left(p^{5}-13 p^{4}+10 p^{3}+350 p^{2}-851 p-2857\right)$ \\
\hline
\end{tabular}

Table 2: $D_{k}(p)=d_{k}^{-1} p^{k}(p+1) A_{k}(p), k=1, \ldots, 9$.

\section{Proof of Theorem 1}

Following some of the arguments in the proof of Lemma 2 in [16], we use (1.1) to rewrite (1.7) as

$$
\widetilde{H}_{n}^{(k)}(q)=(1-q)^{k} \widetilde{G}_{k}(q)
$$

where

$$
\widetilde{G}_{k}(q):=\sum_{j=1}^{p-1} \frac{q^{j}}{\left(1-q^{j}\right)^{k}} .
$$

With $\zeta=e^{2 \pi i / p}$ and using (1.1) again, we have

$$
[p]_{q}=\prod_{m=1}^{p-1}\left(q-\zeta^{m}\right),
$$


and this means that in order to prove (2.4) it suffices to show that

$$
\widetilde{G}_{k}\left(\zeta^{m}\right)=\frac{-1}{p^{k}} \widetilde{D}_{k}(p), \quad m=1,2, \ldots, p-1 .
$$

However, since $p$ is prime, all $\zeta^{m}, m=1,2, \ldots, p-1$, are primitive $p$ th roots of unity, and thus

$$
\widetilde{G}_{k}\left(\zeta^{m}\right)=\sum_{j=1}^{p-1} \frac{\zeta^{m j}}{\left(1-\zeta^{m j}\right)^{k}}=\sum_{j=1}^{p-1} \frac{\zeta^{j}}{\left(1-\zeta^{j}\right)^{k}}=\widetilde{G}_{k}(\zeta) .
$$

Therefore we are done if we can prove (3.2) for $m=1$. We do this by evaluating the coefficients of a certain power series in two different ways.

Lemma 1. For any positive integer $p$ and for $|z|<1$ we have

$$
\frac{p}{1-z^{p}}-\frac{1}{1-z}=\sum_{n=0}^{\infty}(-1)^{n+1} \widetilde{G}_{n+1}(\zeta)(z-1)^{n} .
$$

Proof. It is easy to check that the left-hand side of (3.4) is a holomorphic function for $|z|<1$, and that we have the partial fraction expansion

$$
\frac{p}{1-z^{p}}=\frac{1}{1-z}+\sum_{j=1}^{p-1} \frac{\zeta^{j}}{\zeta^{j}-z} .
$$

Writing $t=\zeta^{j}$ for simplicity, we expand

$$
\frac{t}{t-z}=\frac{t}{t-1} \frac{1}{1-\frac{z-1}{t-1}}=t \sum_{n=0}^{\infty} \frac{(-1)^{n+1}}{(1-t)^{n+1}}(z-1)^{n} .
$$

This, with (3.5) and (3.1), gives (3.4).

The connection with the determinants $\widetilde{D}_{n}(p)$ is given by the next result.

Lemma 2. For $|z|<1$ and a complex parameter $p$ we have

$$
\frac{p}{1-z^{p}}-\frac{1}{1-z}=\sum_{n=0}^{\infty} \frac{(-1)^{n}}{p^{n+1}} \widetilde{D}_{n+1}(p)(z-1)^{n} .
$$

By equating coefficients in (3.4) and (3.6), we immediately obtain (3.2) for $m=1$. This completes the proof of Theorem 1, provided we can prove Lemma 2.

For the proof of Lemma 2 we require a basic recurrence relation for the determinants $\widetilde{D}_{n}(p)$; more properties will be derived in Section 5 . 
Lemma 3. For any real or complex parameter $p$ the determinants $\widetilde{D}_{n}(p)$ satisfy the recurrence relation

$$
\widetilde{D}_{n}(p)=\sum_{j=1}^{n}(-1)^{j-1}\left(\begin{array}{c}
p \\
j+1
\end{array}\right) p^{j-1} \widetilde{D}_{n-j}(p),
$$

where $\widetilde{D}_{0}(p)=1$ by convention. In other words,

$$
\sum_{j=0}^{n}(-1)^{j}\left(\begin{array}{c}
p \\
j+1
\end{array}\right) p^{j} \widetilde{D}_{n-j}(p)=0
$$

Proof. If we expand the determinant $\widetilde{D}_{n}(p)$ (see $(2.1)$ ) by the first column, we see that the minors immediately reduce to $p^{j-1} \widetilde{D}_{n-j}(p)$; this gives (3.7). The identity (3.8) is then obtained by multiplying both sides of $(3.7)$ by $p$.

Proof of Lemma 2. We use the general binomial theorem

$$
z^{p}-1=((z-1)+1)^{p}-1=\sum_{j=1}^{\infty}\left(\begin{array}{l}
p \\
j
\end{array}\right)(z-1)^{j}
$$

where

$$
\left(\begin{array}{l}
p \\
j
\end{array}\right)=\frac{p(p-1) \ldots(p-j+1)}{j !}, \quad j=1,2, \ldots
$$

are the generalized binomial coefficients. Now let

$$
\frac{p}{1-z^{p}}-\frac{1}{1-z}=\widetilde{c}_{0}+\widetilde{c}_{1}(z-1)+\widetilde{c}_{2}(z-1)^{2}+\ldots
$$

and multiply both sides by $z^{p}-1$. Then with (3.9) we get

$$
-p+\sum_{j=1}^{\infty}\left(\begin{array}{l}
p \\
j
\end{array}\right)(z-1)^{j-1}=\sum_{n=0}^{\infty} \widetilde{c}_{n} \sum_{j=1}^{\infty}\left(\begin{array}{l}
p \\
j
\end{array}\right)(z-1)^{n+j}
$$

We now equate coefficients of $(z-1)^{j}, j=0,1,2, \ldots$, and see that for the constant coefficient we have $-p+p=0$ as required, while the other coefficients lead to the system of linear equations

$$
\begin{aligned}
\left(\begin{array}{l}
p \\
2
\end{array}\right) & =\widetilde{c}_{0}\left(\begin{array}{l}
p \\
1
\end{array}\right), \\
\left(\begin{array}{l}
p \\
3
\end{array}\right) & =\widetilde{c}_{0}\left(\begin{array}{l}
p \\
2
\end{array}\right)+\widetilde{c}_{1}\left(\begin{array}{l}
p \\
1
\end{array}\right), \\
\left(\begin{array}{l}
p \\
4
\end{array}\right) & =\widetilde{c}_{0}\left(\begin{array}{l}
p \\
3
\end{array}\right)+\widetilde{c}_{1}\left(\begin{array}{l}
p \\
2
\end{array}\right)+\widetilde{c}_{2}\left(\begin{array}{l}
p \\
1
\end{array}\right),
\end{aligned}
$$


so for any positive integer $n$ we have the matrix equation

$$
\left(\begin{array}{cccc}
\left(\begin{array}{c}
p \\
1
\end{array}\right) & 0 & \ldots & 0 \\
\left(\begin{array}{c}
p \\
2
\end{array}\right) & \left(\begin{array}{c}
p \\
1
\end{array}\right) & \ldots & 0 \\
\vdots & \vdots & \ddots & \vdots \\
\left(\begin{array}{c}
p \\
n
\end{array}\right) & \left(\begin{array}{c}
p \\
n-1
\end{array}\right) & \ldots & \left(\begin{array}{c}
p \\
1
\end{array}\right)
\end{array}\right)\left(\begin{array}{c}
\widetilde{c}_{0} \\
\widetilde{c}_{1} \\
\vdots \\
\widetilde{c}_{n}
\end{array}\right)=\left(\begin{array}{c}
\left(\begin{array}{l}
p \\
2 \\
p \\
3
\end{array}\right) \\
\vdots \\
\left(\begin{array}{c}
p \\
n+1
\end{array}\right)
\end{array}\right) .
$$

If $\widetilde{M}_{n}$ denotes the (lower triangular) matrix on the left, then we can show that its inverse is

$$
\widetilde{M}_{n}^{-1}=\left(\begin{array}{cccc}
p^{-1} & 0 & \cdots & 0 \\
-p^{-2} \widetilde{D}_{1}(p) & p^{-1} & \cdots & 0 \\
p^{-3} \widetilde{D}_{2}(p) & -p^{-2} \widetilde{D}_{1}(p) & \cdots & 0 \\
\vdots & \vdots & \ddots & \vdots \\
(-1)^{n-1} p^{-n} \widetilde{D}_{n-1}(p) & (-1)^{n-2} p^{-n+1} \widetilde{D}_{n-2}(p) & \cdots & p^{-1}
\end{array}\right) .
$$

Indeed, it is obvious that the product of the two matrices is again lower triangular, and that the diagonal elements are 1 . Now, if we multiply the $j$ th column of $A_{n}$ with the $k$ th row of the matrix in (3.13), $1 \leq j<k \leq n$, we obtain

$$
\begin{gathered}
(-1)^{k-j-1} p^{-(k-j)} \widetilde{D}_{k-j-1}(p)\left(\begin{array}{c}
p \\
1
\end{array}\right)+(-1)^{k-j-2} p^{-(k-j)+1} \widetilde{D}_{k-j-2}(p)\left(\begin{array}{l}
p \\
2
\end{array}\right)+ \\
\cdots+p^{-1} \widetilde{D}_{0}(p)\left(\begin{array}{c}
p \\
k-j
\end{array}\right) .
\end{gathered}
$$

By (3.8) with $n=k-j-1$, this last expression vanishes, which shows that the matrix in (3.13) is indeed the inverse of $\widetilde{M}_{n}$ in (3.12).

Finally, we multiply $\widetilde{M}_{n}^{-1}$ with the column vector $((2),(3), \ldots,(n+1))^{T}$, and we get, again with (3.8),

$$
\widetilde{c}_{j}=\frac{(-1)^{j}}{p^{j+1}} \widetilde{D}_{j+1}(p),
$$

and this completes the proof of Lemma 2 .

\section{Proof of Theorem 2}

The proof is similar to that of Theorem 1, and therefore we leave out some of the more obvious details. In analogy to Section 3 we rewrite (1.6) as

$$
H_{n}^{(k)}(q)=(1-q)^{k} G_{k}(q),
$$

where

$$
G_{k}(q):=\sum_{j=1}^{p-1} \frac{1}{\left(1-q^{j}\right)^{k}}
$$


and once again it suffices to show that

$$
G_{k}(\zeta)=\sum_{j=1}^{p-1} \frac{1}{\left(1-\zeta^{j}\right)^{k}}=\frac{(-1)^{k-1}}{p^{k}} D_{k}(-p) .
$$

We begin with the following lemma which is analogous to Lemma 1.

Lemma 4. For any positive integer $p$ and for $|z|<1$ we have

$$
\frac{p z^{p-1}}{1-z^{p}}-\frac{1}{1-z}=\sum_{n=0}^{\infty}(-1)^{n+1} G_{n+1}(\zeta)(z-1)^{n} .
$$

Proof. The only difference to the proof of Lemma 1 lies in the partial fraction expansion

$$
\frac{p z^{p-1}}{1-z^{p}}=\frac{1}{1-z}+\sum_{j=1}^{p-1} \frac{1}{\zeta^{j}-z}
$$

which is again easy to verify.

The following result is analogous to Lemma 2.

Lemma 5. For $|z|<1$ and a complex parameter $p$, we have

$$
\frac{p}{z\left(1-z^{p}\right)}-\frac{1}{1-z}=\sum_{n=0}^{\infty} \frac{(-1)^{n}}{p^{n+1}} D_{n+1}(p)(z-1)^{n} .
$$

If we replace $p$ by $-p$, it is easily seen that the left-hand side of (4.3) becomes the left-hand side of (4.2). Equating coefficients of the powers of $z-1$ in (4.2) and (4.3), we then obtain (4.1). This completes the proof of Theorem 2, provided we can prove Lemma 5.

As in the proof of Lemma 2, we need a recurrence for the determinants in question. We skip the proof which is almost identical to that of Lemma 3.

Lemma 6. For any real or complex parameter $p$ the determinants $D_{n}(p)$ satisfy the recurrence relation

$$
D_{n}(p)=\sum_{j=1}^{n}(-1)^{j-1}\left(\begin{array}{c}
p+1 \\
j+1
\end{array}\right) p^{j-1} D_{n-j}(p),
$$

where $D_{0}(p)=1$ by convention.

Proof of Lemma 5. Using the binomial theorem, we have

$$
z\left(z^{p}-1\right)=((z-1)+1)^{p+1}-(z-1)-1=\sum_{j=0}^{\infty}\left(\begin{array}{c}
p+1 \\
j
\end{array}\right)(z-1)^{j}-(z-1)-1
$$


or

$$
z\left(z^{p}-1\right)=\sum_{j=1}^{\infty}\left(\begin{array}{c}
p+1 \\
j
\end{array}\right)^{*}(z-1)^{j},
$$

where $\left(\begin{array}{c}p+1 \\ 1\end{array}\right)^{*}=p$, while $\left(\begin{array}{c}p+1 \\ j\end{array}\right)^{*}=\left(\begin{array}{c}p+1 \\ j\end{array}\right)$ for $j \geq 2$. Now let

$$
\frac{p}{z\left(1-z^{p}\right)}-\frac{1}{1-z}=c_{0}+c_{1}(z-1)+c_{2}(z-1)^{2}+\ldots,
$$

and multiply both sides by $z\left(z^{p}-1\right)$. Then with (4.5) we get

$$
-p+\sum_{j=1}^{\infty}\left(\begin{array}{c}
p+1 \\
j
\end{array}\right)^{*}(z-1)^{j-1}=\sum_{n=0}^{\infty} c_{n} \sum_{j=1}^{\infty}\left(\begin{array}{c}
p+1 \\
j
\end{array}\right)^{*}(z-1)^{n+j}
$$

As before, equating coefficients of $(z-1)^{j}, j=0,1,2, \ldots$, we obtain for any integer $n$ the matrix equation

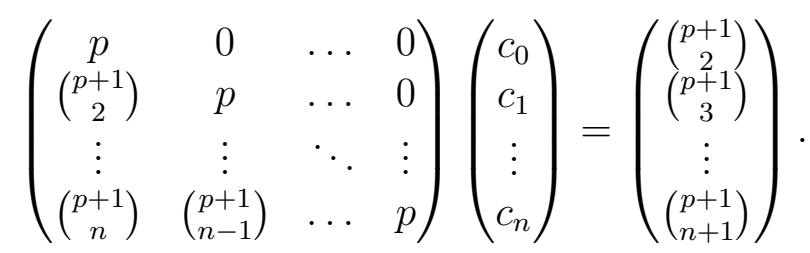

If $M_{n}$ denotes the matrix on the left, then I claim that its inverse is the same as the matrix in (3.13), with $D_{j}(p)$ instead of $\widetilde{D}_{j}(p)$ for all $j$. This can be verified in the same way as in the proof of Lemma 2, using Lemma 6 in this case. Thus we obtain $c_{j}, j=1,2, \ldots, n$, by multiplying the column vectors on the right of (4.7) by the $j$ th row of $M_{n}^{-1}$. Applying Lemma 6 again, we finally get

$$
c_{j}=\frac{(-1)^{j}}{p^{j+1}} D_{j+1}(p)
$$

which holds for all $j \geq 0$ since $n$ is an arbitrary positive integer. This completes the proof of Lemma 5.

\section{$5 \quad$ Further properties of the determinants}

Tables 1 and 2 give rise to some questions and conjectures about the determinants $\widetilde{D}_{n}(p)$ and $D_{n}(p)$ as functions of $p$. In this section we derive a number of properties of these functions.

Proposition 1. (a) The functions $\widetilde{D}_{n}(p)$ are polynomials of degree at most $2 n$.

(b) For $n \geq 1, \widetilde{D}_{n}(p)$ is divisible by $p^{n}$.

(c) For $n \geq 2, p^{-n} \widetilde{D}_{n}(p)$ is a polynomial in $p^{2}$.

(d) For $n \geq 2, p^{-n} \widetilde{D}_{n}(p)$ is divisible by $p^{2}-1$. 
Proof. (a) This follows by induction from (3.7). Indeed, $\widetilde{D}_{1}(p)$ is a polynomial of degree 2. Suppose the statement is true for all $\widetilde{D}_{j}(p)$ for $j \leq n-1$. Then since $\left(\begin{array}{c}p \\ j+1\end{array}\right)$ is a polynomial of degree $j+1$, each term on the right of (3.7) has degree at most $2 n$, which proves the claim.

(b) In each of the $n$ columns in the determinant in (2.1) we can factor $p$ out of all entries.

(c) Given the expression (3.6), it suffices to show that

$$
\frac{p}{1-z^{p}}-\frac{1}{2}(p-1)
$$

is an even function in $p$. But this is easy to verify.

(d) In the first column of the determinant in (2.1) all entries are divisible by $p-1$, and therefore $p^{-n} \widetilde{D}_{n}(p)$ is divisible by $p-1$. By part $(\mathrm{c}), p^{-n} \widetilde{D}_{n}(p)$ is divisible also by $p+1$ for $n \geq 2$.

We continue with some further factorization properties.

Proposition 2. (a) If $p$ is an odd positive integer, then $\widetilde{D}_{n}(p)=0$ for all $n=(2 k+1) p+2$, $k=0,1,2, \ldots$

(b) If $n \geq 3$ is odd and $m$ is a divisor of $n-2$, then $p^{-n} \widetilde{D}_{n}(p)$ is divisible by $p^{2}-m^{2}$. Proof. We combine (3.2) with (3.3) (for $m=1$ ) and rewrite it as

$$
\frac{1}{p^{n}} \widetilde{D}_{n}(p)=-\left(\frac{i}{2}\right)^{n} \sum_{j=1}^{p-1} \frac{e^{(2-n) \pi i j / p}}{\sin ^{n}(\pi j / p)}
$$

When $n \equiv p+2(\bmod 2 p)$, then

$$
e^{(2-n) \pi i j / p}=e^{\pi i j}=(-1)^{j}
$$

so the sum in $(5.1)$ becomes

$$
\sum_{j=1}^{p-1} \frac{(-1)^{j}}{\sin ^{n}(\pi j / p)}
$$

which vanishes for odd $p \operatorname{since} \sin (\pi j / p)=\sin (\pi(p-j) / p)$ for $j=1,2, \ldots, p-1$, and $j \neq p-j$ for odd $p$.

To prove (b), we note that by (a), $m$ is a zero of $\widetilde{D}_{n}(p)$, so it is divisible by $p-m$. By Proposition $1(\mathrm{c})$ it is also divisible by $p+m$.

As an illustration of this last result see, e.g., the case $k=11$ in Table 1.

The next result uses the Bernoulli numbers $B_{n}$ which can be defined by the generating function

$$
\frac{x}{e^{x}-1}=\sum_{n=0}^{\infty} B_{n} \frac{x^{n}}{n !}, \quad|x|<2 \pi ;
$$


for properties and further details see, e.g., [10, p. 283 ff.]. One property we are going to use below is the most basic recurrence relation

$$
\sum_{j=0}^{n}\left(\begin{array}{c}
n+1 \\
j
\end{array}\right) B_{j}=0, \quad n \geq 1 .
$$

Along with $B_{0}=1$, this allows for a quick evaluation of the first few values $B_{1}=-\frac{1}{2}, B_{2}=$ $\frac{1}{6}, B_{4}=-\frac{1}{30}, B_{6}=\frac{1}{42}, B_{8}=-\frac{1}{30}, B_{10}=\frac{5}{66}, B_{12}=-\frac{691}{2730}$, and it can be shown that $B_{2 k+1}=0$ for all $k \geq 1$.

Proposition 3. The coefficient of $p^{2 n}$ in the polynomial $\widetilde{D}_{n}(p)$ is $\frac{1}{n !} B_{n}$. For even $n \geq 2$, this is also the leading coefficient of $\widetilde{D}_{n}(p)$.

Proof. Let $a_{n}$ be the coefficient in question. Then, since the leading coefficient in the polynomial $\left(\begin{array}{c}p \\ j+1\end{array}\right) p^{j-1}$ is $1 /(j+1)$ ! for all $j$, by equating coefficients of $p^{2 n}$ in the recurrence relation $(3.8)$ we get

$$
\sum_{j=0}^{n}(-1)^{j} \frac{1}{(j+1) !} a_{n-j}=0 .
$$

Multiplying both sides by $(-1)^{n}(n+1)$ ! and changing the order of summation, we obtain

$$
\sum_{j=0}^{n}(-1)^{j}\left(\begin{array}{c}
n+1 \\
j
\end{array}\right) j ! a_{j}=0 .
$$

Recall that $\widetilde{D}_{0}(p)=1$ by convention. Hence, comparing (5.5) and (5.3), we get $(-1)^{j} j ! a_{j}$ $=B_{j}$ for all $j \geq 1$, and in particular $a_{j}=B_{j} / j$ ! for all $j \geq 2$ since $B_{2 k+1}=0$ for $k \geq 1$. This proves both statements of the proposition.

This last proof also shows that the coefficient of $p^{2}$ in $\widetilde{D}_{1}(p)$ is $-B_{1}=\frac{1}{2}$. This is consistent with $\widetilde{D}_{1}(p)=p(p-1) / 2$, and explains why $\widetilde{D}_{1}(p)$ is the only odd-index determinant $\widetilde{D}_{n}(p)$ that has full degree $2 n$.

Table 2 indicates that the polynomials $D_{n}(p)$ have properties similar to those of the $\widetilde{D}_{n}(p)$, with the main difference that $p^{-n} D_{n}(p)$ is not an even or an odd function in $p$. I therefore omit the details of the proofs of the following three results.

Proposition 4. (a) The functions $D_{n}(p)$ are polynomials of degree at most $2 n$.

(b) For $n \geq 1, D_{n}(p)$ is divisible by $p^{n}$.

(c) For $n \geq 1, p^{-n} D_{n}(p)$ is divisible by $p+1$.

For part (a) we can use the recurrence relation (4.4), and (b), (c) follow immediately from (2.5). The next result, which is analogous to Proposition 2, uses (4.1).

Proposition 5. If $n \geq 1$ is odd and $m$ is a divisor of $n$, then $p^{-n} D_{n}(p)$ is divisible by $p+m$.

As an illustration of this, see the case $k=9$ in Table 2. 
Proposition 6. The coefficient of $p^{2 n}$ in $D_{n}(p)$ is $\frac{1}{n !} B_{n}$, which is also the leading coefficient of $D_{n}(p)$ for even $n \geq 2$.

The proof of this result is almost identical to that of Proposition 3 since the recurrence relation (4.4) leads to the same relation (5.4) as before for the coefficients in question.

We have seen that $D_{n}(p)$ is neither an even nor an odd function of $p$. However, we have the following property; it is not difficult to obtain by manipulating the generating function (4.3).

Proposition 7. For all $n \geq 1$ we have

$$
(-1)^{n}\left(D_{n}(-p)-\frac{1}{2}(-p)^{n+1}\right)=D_{n}(p)-\frac{1}{2} p^{n+1} .
$$

The two determinant sequences studied in this paper are connected by the following two easy identities.

Proposition 8. For all $n \geq 1$ we have

$$
\widetilde{D}_{n}(p)=D_{n}(p)-p D_{n-1}(p)
$$

and

$$
p^{-n} D_{n}(p)=\sum_{m=0}^{n} p^{-m} \widetilde{D}_{m}(p)
$$

where $D_{0}(p)=\widetilde{D}_{0}(p)=1$.

Proof. We use the simple identity

$$
\frac{p}{1-z^{p}}=((z-1)+1) \frac{p}{z\left(1-z^{p}\right)}
$$

with (3.11) and (4.6). Then, equating coefficients of the powers of $z-1$, we easily obtain

$$
\widetilde{c}_{n}=c_{n}+c_{n-1}
$$

for $n \geq 0$, with $c_{-1}=-1$. The identity (5.7) then follows from (3.14) and (4.8). Finally we replace $n$ by $m$ in (5.7), multiply both sides by $p^{-m}$ and sum from $m=0$ to $m=n$. This sum "telescopes", giving us (5.8).

Remark 1. The identity (5.8) could be derived directly, using a slight variant of (5.9). Also, (5.7) could be derived directly from the defining determinants (2.1) and (2.5) as follows. In the determinant (2.1), add the second column to the first, the third column to the second, etc., until the last column is added to the second-last one, always using the identity $\left(\begin{array}{c}p \\ j+1\end{array}\right)+\left(\begin{array}{c}p \\ j\end{array}\right)=\left(\begin{array}{c}p+1 \\ j+1\end{array}\right)$. This does not change the determinant, but gives us a new matrix that is identical to the one in (2.5) with the exception of the lower right entry which remains $\left(\begin{array}{c}p \\ 2\end{array}\right)$ instead of $\left(\begin{array}{c}p+1 \\ 2\end{array}\right)$. Using the summation rule for determinants, we note that $D_{n}(p)-\widetilde{D}_{n}(p)$ is the determinant of a similar matrix again, only this time 
with $\left(\begin{array}{c}p+1 \\ 2\end{array}\right)-\left(\begin{array}{l}p \\ 2\end{array}\right)=p$ in the lower right corner as the only nonzero entry in that column. Expanding along this last column, the determinant is obviously $p D_{n-1}(p)$, and this proves (5.7).

Remark 2. Returning to the generalized $q$-harmonic numbers of Sections 1 and 2, we rewrite (1.6) as

$$
H_{p-1}^{(k)}(q):=\sum_{j=1}^{p-1} \frac{1-q^{j}+q^{j}}{[j]_{q}^{k}}=(1-q) \sum_{j=1}^{p-1} \frac{1}{[j]_{q}^{k-1}}+\sum_{j=1}^{p-1} \frac{q^{j}}{[j]_{q}^{k}},
$$

so that with (1.7) we have

$$
H_{p-1}^{(k)}(q)=(1-q) H_{p-1}^{(k-1)}(q)+\widetilde{H}_{p-1}^{(k)}(q)
$$

and (2.6) gives

$$
\widetilde{H}_{p-1}^{(k)}(q) \equiv \frac{(-1)^{k-1}}{p^{k}}\left(D_{k}(-p)+p D_{k-1}(-p)\right)(1-q)^{k} \quad\left(\bmod [p]_{q}\right) .
$$

Now we use (5.7) and the fact that $p^{-k} \widetilde{D}_{k}(p)$ is an even function, and obtain (2.4). This is an alternative proof of Theorem 1, provided that Theorem 2 has been proven.

On the other hand, by iterating (5.10) we immediately obtain

$$
H_{p-1}^{(k)}(q)=(1-q)^{k}(p-1)+\sum_{j=1}^{k}(1-q)^{k-j} \widetilde{H}_{p-1}^{(j)}(q) .
$$

Now, if we use the congruence (2.4), then (5.8), and finally the identity $D_{k}(p)-p^{k+1}=$ $(-1)^{k} D_{k}(-p)$, which follows directly from (5.6), then we obtain (2.6). This shows that Theorem 1 implies Theorem 2.

\section{Degenerate Bernoulli Numbers}

In addition to the coefficient of $p^{2 n}$ of the polynomial $p^{-n} \widetilde{D}_{n}(p)$, which was determined in Proposition 3, we can just as easily find the constant coefficient of this polynomial. Let $\widetilde{b}_{n}$ be this coefficient. Multiply both sides of $(3.7)$ by $-(z-1)$, and set $x=z-1$. Then we have

$$
\lim _{p \rightarrow 0} \frac{p x}{(1+x)^{p}-1}=1+\sum_{n=1}^{\infty}(-1)^{n} \widetilde{b}_{n} x^{n},
$$

and l'Hospital's rule gives

$$
\frac{x}{\log (1+x)}=1+\sum_{n=1}^{\infty}(-1)^{n} \widetilde{b}_{n} x^{n} .
$$

Now, the left-hand side of (6.1) is known as the generating function of the Bernoulli numbers of the second kind; see, e.g., [12, p. 265 ff.]. Hence we have proved 
Proposition 9. For $n \geq 1$ the constant coefficient of the polynomial $p^{-n} \widetilde{D}_{n}(p)$ is $(-1)^{n} b_{n}$, where $b_{n}$ is the nth Bernoulli number of the second kind.

The first few values of the $b_{n}$ are $b_{0}=1, b_{1}=1 / 2, b_{2}=-1 / 2, b_{3}=1 / 24, b_{4}=-19 / 720$, $b_{5}=3 / 160, b_{6}=-863 / 60480$. This is consistent with Table 1 .

The question now arises whether there are explicit expressions also for the other coefficients of the polynomials $p^{-n} \widetilde{D}_{n}(p)$. This is indeed the case, and there is a close relationship with the degenerate Bernoulli numbers. These numbers (in fact, polynomials) were first studied by Carlitz [3], and can be defined by the generating function

$$
\frac{x}{(1+\lambda x)^{1 / \lambda}-1}=\sum_{n=0}^{\infty} \beta_{n}(\lambda) \frac{x^{n}}{n !} .
$$

Carlitz [3], [4] proved numerous properties, including the fact that $\beta_{n}(\lambda)$ is a polynomial in $\lambda$ of degree $\leq n$. By comparing (6.2) with (5.2) it is clear that

$$
\lim _{\lambda \rightarrow 0} \beta_{n}(\lambda)=B_{n}
$$

The following result gives a surprising connection with the determinants $\widetilde{D}_{n}(p)$.

Theorem 3. For all $n \geq 0$ we have

$$
\beta_{n}(\lambda)=(-1)^{n} n ! \lambda^{2 n} \widetilde{D}_{n}\left(\frac{1}{\lambda}\right),
$$

or equivalently

$$
p^{-n} \widetilde{D}_{n}(p)=\frac{(-1)^{n}}{n !} p^{n} \beta_{n}\left(\frac{1}{p}\right) .
$$

That is, the polynomials $\beta_{n}(\lambda)$ and $p^{-n} \widetilde{D}_{n}(p)$ are, up to a constant factor, reciprocal to each other.

Proof. As in the proof of Proposition 9 we start with (3.7), multiply both sides by $-(z-1)$, and set $y=z-1$. With the convention $\widetilde{D}_{0}(p)=1$ we then have

$$
\frac{p y}{(1+y)^{p}-1}=\sum_{n=0}^{\infty} \frac{(-1)^{n}}{p^{n}} \widetilde{D}_{n}(p) y^{n} \text {. }
$$

Finally we set $p=1 / \lambda$ and $y=x \lambda$; then (6.6) compared with (6.2) gives (6.4). The identity (6.5) is obvious, with $\lambda=1 / p$.

Theorem 3 implies that all the properties of one the set of polynomials carry over to the other. For instance, Proposition 2(b) immediately gives the following, apparently new, divisibility property of the polynomial $\beta_{n}(\lambda)$.

Corollary 1. If $n \geq 3$ is odd and $m$ is a divisor of $n-2$, then $m^{2} \lambda^{2}-1$ divides $\beta_{n}(\lambda)$. 
In one of the more recent papers on degenerate Bernoulli numbers, Howard [11] found a remarkably simple explicit expression for the coefficients of the $\beta_{n}(\lambda)$, namely

Theorem 4 (Howard). For $n \geq 2$ we have

$$
\beta_{n}(\lambda)=n ! b_{n} \lambda^{n}+\sum_{j=1}^{\lfloor n / 2\rfloor} \frac{n}{2 j} B_{2 j} s(n-1,2 j-1) \lambda^{n-2 j},
$$

where $s(n, r)$ is the Stirling number of the first kind and $b_{n}$ the Bernoulli number of the second kind.

Recall that the Bernoulli numbers of the second kind are defined by the generating function (6.1). For properties of Stirling numbers see, e.g., [6] or, in a different notation, [10]. Combining (6.7) with (6.5), we get the following immediate consequence.

Corollary 2. For $n \geq 2$ we have

$$
p^{-n} \widetilde{D}_{n}(p)=(-1)^{n} b_{n}+\frac{(-1)^{n}}{(n-1) !} \sum_{j=1}^{\lfloor n / 2\rfloor} \frac{B_{2 j}}{2 j} s(n-1,2 j-1) p^{2 j} .
$$

It is obvious that (6.8) contains both Proposition 3 and Proposition 9 as special cases. Let now $n=2 k+1, k \geq 1$. Then $\lfloor n / 2\rfloor=k$, and the leading coefficient of (6.8) becomes

$$
\frac{-1}{(2 k) !} \frac{B_{2 k}}{2 k} s(2 k, 2 k-1) \text {. }
$$

Since $s(n, n-1)=-n(n-1) / 2$ (see, e.g., [6, p. 214]), we get the following result supplementing Propostion 3.

Corollary 3. For all $k \geq 1$, the polynomials $p^{-(2 k+1)} \widetilde{D}_{2 k+1}(p)$ have degree $2 k$, with leading coefficient

$$
\frac{2 k-1}{2} \frac{B_{2 k}}{(2 k) !}
$$

Finally, we can use Corollary 2, and in fact the partial results Proposition 3 and Proposition 9, to derive two known classical determinant expressions. First, in (2.1) with $n$ in place of $k$, we divide each column by $p$. Then we can see from (3.10) that upon setting $p=0$ each entry $\left(\begin{array}{l}p \\ j\end{array}\right)$ becomes $(-1)^{j-1} / j$. We then eliminate the minus signs, which are arranged in checker-board fashion, by first multiplying columns $1,3,5, \ldots$, and then rows $2,4, \ldots$, by -1 , for a total of $(-1)^{n}$. Altogether, if we set $p=0$ in (6.8), we obtain

$$
b_{n}=\operatorname{det}\left(\begin{array}{ccccc}
\frac{1}{2} & 1 & 0 & \ldots & 0 \\
\frac{1}{3} & \frac{1}{2} & 1 & \ldots & 0 \\
\vdots & \vdots & \vdots & \ddots & \vdots \\
\frac{1}{n} & \frac{1}{n-1} & \frac{1}{n-2} & \ldots & 1 \\
\frac{1}{n+1} & \frac{1}{n} & \frac{1}{n-1} & \ldots & \frac{1}{2}
\end{array}\right) \text {. }
$$


This determinant can be found in an old paper by Glaisher [7].

Next, the identities (6.3) and (6.4) give

$$
B_{n}=(-1)^{n} n ! \lim _{p \rightarrow \infty} p^{-2 n} \widetilde{D}_{n}(p) .
$$

Note that the $(j, k)$-th entry in (2.1) is a polynomial in $p$ of degree $j-k+2$ if this number is positive, and vanishes otherwise. Since a determinant is a sum or difference of products of exactly one element in each row and each column, the $j$ and $k$ cancel each other out as contributions to the degree, and so each nonzero product has degree exactly $p^{2 n}$. Thus, if we divide each entry $\left(\begin{array}{c}p \\ j\end{array}\right)$ by $p^{j}$, this amounts to dividing the entire determinant $\widetilde{D}_{n}(p)$ by $p^{2 n}$. Taking the limit as $p \rightarrow \infty$, the relevant entries then become $1 / j$ !, and we get with (6.9),

$$
B_{n}=(-1)^{n} n ! \operatorname{det}\left(\begin{array}{ccccc}
\frac{1}{2 !} & 1 & 0 & \ldots & 0 \\
\frac{1}{3 !} & \frac{1}{2 !} & 1 & \ldots & 0 \\
\vdots & \vdots & \vdots & \ddots & \vdots \\
\frac{1}{n !} & \frac{1}{(n-1) !} & \frac{1}{(n-2) !} & \ldots & 1 \\
\frac{1}{(n+1) !} & \frac{1}{n !} & \frac{1}{(n-1) !} & \ldots & \frac{1}{2 !}
\end{array}\right) \text {, }
$$

a determinant that can be found in [7] as well, and also in [15].

These last two determinant expressions can therefore be seen as special cases of the determinant in (2.1), via Theorem 3.

\section{References}

[1] G. E. Andrews, q-analogs of the binomial coefficient congruences of Babbage, Wolstenholme and Glaisher, Discrete Math. 204 (1999), 15-25.

[2] G. E. Andrews, R. Askey, and R. Roy, Special Functions, Encyclopedia of Mathematics and Its Applications 71, Cambridge University Press, Cambridge, 1999.

[3] L. Carlitz, A degenerate Staudt-Clausen theorem, Arch. Math. 7 (1956), 28-33.

[4] L. Carlitz, Degenerate Stirling, Bernoulli and Eulerian numbers, Utilitas Math. 15 (1979), 51-88.

[5] G.-S. Cheon and M. E. A. El-Mikkawy, Generalized harmonic number identities and a related matrix representation, J. Korean Math. Soc. 44 (2007), 487-498.

[6] L. Comtet, Advanced Combinatorics, Reidel, Dordrecht, 1974.

[7] J. W. L. Glaisher, Expressions for Laplace's coefficients, Bernoullian and Eulerian numbers, ${ }^{6} c$, as determinants, Messenger Math. 6 (1877), 49-63.

[8] J. W. L. Glaisher, On the residues of the sums of products of the first $p-1$ numbers and their powers, to modulus $p^{2}$ or $p^{3}$, Quart. J. Math. 31 (1900), 321-353.

[9] J. W. L. Glaisher, On the residues of the sums of the inverse powers of numbers in arithmetical progression, Quart. J. Math. 32 (1900), 271-288. 
[10] R. L. Graham, D. E. Knuth, and O. Patashnik, Concrete Mathematics, 2nd edition, Addison-Wesley Publ. Co., Reading, MA, 1994.

[11] F. T. Howard, Explicit formulas for degenerate Bernoulli numbers, Discrete Math. 162 (1996), 175-185.

[12] C. Jordan, Calculus of Finite Differences, 2nd edition, Chelsea, New York, 1947,

[13] E. Lehmer, On congruences involving Bernoulli numbers and the quotients of Fermat and Wilson, Ann. of Math. Oxford Ser. 39 (1938), 350-360.

[14] R. J. McIntosh, On the converse of Wolstenholme's theorem, Acta Arith. 71 (1995), 381-389.

[15] A. Sachse, Ueber die Darstellung der Bernoulli'schen und Euler'schen Zahlen durch Determinanten, Arch. Math. Physik 68 (1882), 427-432.

[16] L.-L. Shi and H. Pan, A q-analogue of Wolstenholme's harmonic series congruence, Amer. Math. Monthly 114 (2007), 529-531.

[17] J. Wolstenholme, On certain properties of prime numbers, Quart. J. Math. Oxford Ser. 5 (1862), 35-39. 